Word count:3,031

Tables:2

Figures:1

\title{
Frontal lobe Volumes in Schizophrenia: Effects of Stage and Duration of Illness
}

Preethi Premkumar $^{{ }^{*}}$; Veena Kumari ${ }^{\mathrm{a}}$; Dominic Fannon, Philip J. Corr ${ }^{\mathrm{b}}$; Tonmoy Sharma ${ }^{\mathrm{c}}$

${ }^{a}$ Department of Psychology, PO78, Institute of Psychiatry, King's College London, London, UK

${ }^{\mathrm{b}}$ Department of Psychology, University of Wales, Swansea, UK

${ }^{c}$ Clinical Neuroscience Research Centre, Dartford, Kent, UK

*Corresponding author

Preethi Premkumar

Department of Psychology

Institute of Psychiatry

De Crespigny Park

London SE5 8AF

Tel: +44 2078480233

Fax: +44 2078480860

E.mail: Preethi.premkumar@iop.kcl.ac.uk 


\begin{abstract}
While the changes in the volume of the temporal lobe and its sub-regions over the course of illness have been studied in patients with schizophrenia, few studies have examined changes in the frontal lobe between the first episode and the chronic stage. In this study, we focussed on the effect of illness stage and duration of illness on the volume of frontal lobe regions, though we examined several other regions to establish the specificity of any effects observed for this region. We compared the volumes of brain regions among 34 first-episode schizophrenia patients, 49 chronic schizophrenia patients, 18 healthy controls matched, on average, to the first-episode patients and 21 healthy controls matched, on average, to the chronic patients. Logarithmic regression analyses examined the relationships between the duration of illness and the brain regional volumes in the patient group. The results showed that chronic patients had smaller prefrontal cortical grey matter volumes, but larger premotor cortical volumes compared to first-episode patients and matched healthy controls; they also had smaller parieto-occipital grey matter volumes and larger putamen and lateral ventricles. There was a significant exponential relation between the duration of illness and the volumes of these regions. The exponential relation suggests that the prefrontal cortex, premotor cortex, parieto-occipital cortex and putamen are susceptible to change as the disorder persists. The larger volumes of the premotor cortices in the chronic relative to the first-episode patients may reflect a compensatory mechanism of the premotor cortex following loss of function of the prefrontal cortex. Alternately, the enlargement of the premotor cortex may be secondary to that of the putamen, since brain enlargement in schizophrenia has been typically reported in the basal ganglia as a result of antipsychotic medication.
\end{abstract}

Keywords: Schizophrenia, progression, duration of illness, magnetic resonance imaging, frontal lobe, temporal lobe 


\section{Introduction}

The idea that schizophrenia is a progressive illness associated with a neurodegenerative process has existed since the illness was first described (Kraepelin, 1899/1989; 1919/1971). However, the validity of this concept remains controversial, since there is evidence that both supports and appears inconsistent with this idea. Absence of clear neuropathologic changes consistent with a neurodegenerative process (Pakkenberg, 1993; Selemon et al, 1998;

Weinberger \& McClure, 2002), along with the fact that schizophrenia is characterized by abnormalities of brain structure that are present from the early stages of the illness (Copolov et al, 2000) are among the lines of evidence that support the hypothesis that schizophrenia is instead a neurodevelopmental process (Weinberger \& McClure, 2002). It is, however, still uncertain as to whether structural abnormalities are static or progressive (Harrison, 1999; Stevens, 1991; Woods, 1998).

The evidence for whether ventricular enlargement is static or progressive is contradictory. Some longitudinal studies have demonstrated progressive ventricular enlargement (DeLisi et al, 1998; 2004; 1997; 1995; Leiberman et al, 2001; Mathalon et al, 2001; Nair et al, 1997; Saijo et al, 2001), whereas others failed to detect progression (James et al, 2002; Jaskiw et al, 1994) or detected progression only in a subpopulation of studied patients (Nair et al, 1997; Puri et al, 2001). DeLisi et al (2004) observed that ventricular enlargement was greater during the second half of a ten-year follow-up. Particular changes in brain morphology may shed light on the neurodevelopmental versus neurodegenerative debate (Woods, 1998). Woods et al (1998; 1996) found that the frontal intracranial volume was significantly smaller and the brain tissue-to-cranial 
volume ratio was lower in chronic schizophrenia patients than healthy controls. It was inferred that while the decrease in frontal intracranial volume was due to a pathological process that occurred before brain growth was complete, the generalized reduction in brain volume to cranial volume ratio was due to a process that affected the whole cerebrum after brain growth was complete. Gur et al (1998) reported greater reduction in temporal lobe volume in first-episode than previously-treated patients over a 2.5 year period. However, DeLisi and Hoff (2005) failed to find a decrease in temporal lobe volume in first-episode patients over a ten-year period.

The longitudinal course of the frontal lobe in schizophrenia has been examined by four published studies using a regions of interest approach (Bachmann et al, 2004; Dickey et al, 2004; Gur et al, 1998; (Molina et al, 2004). Three of these studies examined the longitudinal course of frontal lobe volume (Bachmann et al, 2004; Dickey et al, 2004) while one study compared frontal lobe volumes of first-episode and chronic schizophrenia patients (Molina et al, 2004). Bachmann et al (2004) reported significant decreases in left and right frontal lobe volumes in 14 first-episode patients over a 14-month period, while Gur et al (1998) observed greater left than right hemisphere reduction in the frontal lobe in 40 schizophrenia patients over a 2.5 year period. Furthermore, the left hemispheric reduction was greater in first-episode patients than previouslytreated patients. Dickey et al (2004) failed to observe a change in prefrontal volumes in 12 schizophrenia patients over a 1.5 year follow-up period. The follow-up interval may be a critical factor in longitudinal studies with significant changes detected in studies only with 1.5 year or longer follow up. Molina et al (2004) compared schizophrenia patients from two illness stages. A volumetric decrease in prefrontal cortex (PFC) grey matter, while absent in first- 
episode patients, was present in short-term and long-term chronic schizophrenia patients. The present study compared brain regional volume in patients with first-episode schizophrenia, chronic schizophrenia and healthy controls that were matched, on average, to patients at each illness stage. Although our focus was on the frontal lobe, we examined the volumes of a large number of brain regions of interest. We also investigated the relationship between duration of illness (DoI) and frontal lobe volume. We hypothesized, based on the findings of Molina et al. (2004), that (a) chronic patients would have smaller brain volumes compared with first-episode patients, and (b) there would be an exponential relation between DoI and brain volume across the patient groups.

\section{Methods}

\subsection{Participants}

Two studies were conducted to examine brain structural differences between individuals with schizophrenia and healthy controls. The first study comprised 34 first-episode (FE) patients with schizophrenia as determined by the Structured Clinical Interview for DSM-I (Spitzer et al, 1995 ) and 18 healthy controls (findings from this cohort have been included in previous publications, Ettinger et al, 2002; Fannon et al, 2000; Sumich et al, 2002). Diagnosis of schizophrenia in all first-episode cases was re-confirmed after 1 year. Ten patients were antipsychotic naïve, 17 were receiving conventional antipsychotics and seven were receiving atypical antipsychotics. None of the patients had had more than 12 weeks of antipsychotic 
medication. The second study comprised 49 patients with a DSM-IV diagnosis of schizophrenia and 21 healthy controls. Patients were included if they had been receiving treatment with conventional antipsychotics for at least 28 days prior to admission to the study and were aged 1865 years. The description of the sample as having chronic schizophrenia was based on the patients having a DoI greater than 3 years (range 3 to 37 years). Exclusion criteria (for both studies) were a positive urine drug screen test, alcohol consumption in the 24 hours prior to scanning. Exclusion criteria for controls, in addition to those applied to the patients, were presence of a personal history of an axis disorder and a family history of psychosis warranted exclusion. Diagnostic assessment using the non-patient version of the Structured Clinical Interview for DSM-IV (SCID) (First et al, 1996b) were performed by research psychiatrists on controls.

\subsection{Image acquisition}

MRI scans were acquired at the Maudsley Hospital, London using a 1.5-T G.E. Signa system. A series of sagittal and axial scout views were acquired to correct for head tilt and to localize imaging coordinates. For participants from the first-episode study, the following sequence was used: repetition time (TR) 11.3 milliseconds, time to inversion (TI) 300 milliseconds, echo time (TE)_ 2.2 milliseconds, flip angle_ $20^{\circ}$, number of excitations (NEX) 1, producing 124 1.5-mm thick axial slices covering the entire brain.. For participants in the chronic study, the following sequence was used $\left(\mathrm{TR}=35 \mathrm{~ms}, \mathrm{TE}=5 \mathrm{~ms}\right.$, flip angle $35^{\circ}$, matrix $=$ $256 \times 256 \times 124)$. 


\subsection{Measurement of brain regions}

Stereological measurements using the software, MEASURE (Barta et al, 1997), followed existing criteria for the following brain regions of interest (ROIs): whole brain, cortical grey matter, lateral ventricles and temporal lobe (DeLisi et al, 1995), hippocampus (Stephanis et al, 1999), thalamus (Portas et al, 1998), putamen and cerebellum. The measurement of the putamen was performed in the coronal and axial views, though the coronal view was mainly used for the rating since the ROI was present across more number of slices compared to the axial view, thereby allowing greater precision. The putamen was separated from the caudate nucleus by the internal capsule and separated from the claustrum more ventrally by the external capsule. On the coronal slices before the appearance of the globus pallidus, the most distinct appearance of the internal capsule was used as a guide to determine the last horizontal line of pixels to be counted for that slice. In the more ventral slices, the pixels in the putamen that were contiguous with the temporal pole were excluded from the measurement. The cerebellum was seen as a distinct structure consisting of four lobes that were separate from the cerebral cortex and brain stem. The cortex itself was divided into 4 sub-regions based on cytoarchitectonic boundaries: prefrontal, premotor, sensorimotor and parieto-occipital ROIs (Bilder et al, 1994). Separate total and grey matter ROIs for cortical sub-regions led to 17 ROIs. Ratings were performed by researchers, including one of the authors (P.P.), blind to participant diagnosis. The raters were trained in rating ROIs on 10 random scans from the two studies until an inter-rater reliability $\geq 0.9$ was achieved.

\subsection{Statistical analysis}


Due to the lack of homogeneity of variance between the four participant groups, we performed non-parametric Kruskal-Wallis $\mathrm{H}$ tests for age and education. Chi-square tests were performed for gender and handedness.

To test the hypothesis that brain volume is lower in chronic schizophrenia compared with first-episode schizophrenia and healthy controls, we performed a multivariate analysis of variance (MANOVA; Wilks' F) on 15 regions. To test whether the slope of the difference between $\mathrm{FE}$ and $\mathrm{CH}$ patients differed significantly from that of the difference between $\mathrm{FE}$ and $\mathrm{CH}$ controls, we specified an interaction term: (participant type: patient versus control) by (illness stage: first-episode versus chronic). Heterogeneity of variance meant that Wilcoxon Signed Ranks test was performed for hippocampus and putamen.

The relation between DoI and brain regional volumes was tested using a logarithmic regression analysis because it was expected that brain volume change over time would not be linear (Molina et al, 2004) and because of variance heterogeneity in DoI between patient groups.

All statistical analyses were performed using the Statistical Package for Social Sciences, version 10.1.3. 


\section{Results}

As expected and shown in Table 1, CH patients and controls were older than FE patients and controls. Patients had fewer years in education than controls (Table 1).

\section{Tables 1 and 2 about here}

\subsection{Brain region comparisons}

The participant type-by-illness stage interaction was significant $(\mathrm{F}=2.98, \mathrm{df}=15,106, p$ $=0.001)$. Patients and controls differed in brain volume; and in turn, first-episode patients differed from chronic patients. The effect was strongest in the grey matter of prefrontal cortex as shown in Table 2. Post hoc Scheffe tests revealed that the difference in PFC grey matter volume was greater between patient groups than between control groups. The $\mathrm{CH}$ patients had smaller volumes than FE patients (mean difference $=13.76$, S.E. $=2.54,95 \%$ C.I., 20.97 to 6.56 ), $\mathrm{FE}$ controls (mean difference $=17.47$, S.E. $=3.14,95 \%$ C.I., 26.37 to 8.57 ) and CH controls (mean difference $=10.57$, S.E. $=2.97,95 \%$ C.I., 18.99 to 2.15 ). This pattern was also seen in total prefrontal cortex, with $\mathrm{CH}$ patients having smaller volumes than $\mathrm{FE}$ patients (mean difference $=$ 13.43, S.E. $=4.21,95 \%$ C.I., 25.35 to 1.5$)$, FE controls (mean difference $=20.54$, S.E. $=5.19$, 95\% C.I., 35.27 to 5.81 ) and $\mathrm{CH}$ controls (mean difference $=14.43$, S.E. $=4.91$, $95 \%$ C.I., 28.36 to 0.49 ). Post hoc analyses for grey matter of parieto-occipital cortex also revealed a similar pattern of $\mathrm{CH}$ patients having smaller volumes than the other 3 groups: $\mathrm{CH}$ patients vs. FE patients (mean difference $=18.22$, S.E. $=5.23,95 \%$ C.I., 33.06 to 3.38$), \mathrm{CH}$ patients vs. $\mathrm{FE}$ controls (mean difference $=36.24$, S.E. $=6.46,95 \%$ C.I., 54.57 to 17.92 ) and $\mathrm{CH}$ patients vs. 
$\mathrm{CH}$ controls (mean difference $=23.72$, S. E. $=6.11,95 \%$ C.I., 41.06 to 6.38). No significant differences were observed between FE patients and FE controls in the volumes of these regions.

The effect was reversed for lateral ventricles, total premotor cortex and putamen. In the lateral ventricles, $\mathrm{CH}$ patients had larger volumes than $\mathrm{FE}$ patients (mean difference $=3.62$, S.E. $=1.13,95 \%$ C.I., 0.44 to 6.81 ), FE controls (mean difference $=5.07$, S.E. $=1.39,95 \%$ C.I., 1.14 to 9) and $\mathrm{CH}$ controls (mean difference $=4.03$, S.E. $=1.31,95 \%$ C.I., 0.3 to 7.75 ). The premotor cortical volume was larger in $\mathrm{CH}$ patients only in comparison with FE patients (mean difference $=18.43$, S.E. $=6.09,95 \%$ C.I., 1.17 to 35.71$)$. The mean volumes of the putamen also suggest that $\mathrm{CH}$ patients had larger volume than the other groups and that it was unlikely that there was a significant difference among the other three groups.

\section{Figure 1 about here}

\subsection{Relationship between duration of illness and brain volumes in patients}

There was a significant log-linear association between DoI and PFC grey matter $\left(\mathrm{R}^{2}=\right.$ $0.22, \mathrm{~F}=22.86, \mathrm{df}=81, \mathrm{P}<0.001)$, such that the $\mathrm{PFC}$ grey matter volume decreased more rapidly during the initial years of illness and slowed down in later years (Figure 1a). In a similar manner, we found a significant association between DoI and grey matter of parieto-occipital cortex $\left(\mathrm{R}^{2}=0.11, \mathrm{~F}=9.49, \mathrm{df}=81, \mathrm{P}=0.003\right)$, total prefrontal cortex $\left(\mathrm{R}^{2}=0.11, \mathrm{~F}=9.48, p=\right.$ $0.003)$, thalamus $\left(\mathrm{R}^{2}=0.10, \mathrm{~F}=9.33, \mathrm{df}=81, p=0.003\right)$ and cortical grey matter $\left(\mathrm{R}^{2}=0.08, \mathrm{~F}=\right.$ 7.27, $\mathrm{df}=81, p=0.009$ ) (Figure1, b-e). The direction of this relation was reversed between DoI and total premotor cortex $\left(\mathrm{R}^{2}=0.07, \mathrm{~F}=6.14, \mathrm{df}=81, p=0.02\right)$, DoI and lateral ventricles $\left(\mathrm{R}^{2}=\right.$ 
$0.05, \mathrm{~F}=4.14, \mathrm{df}=81, p=0.045)$, and DoI and putamen $\left(\mathrm{R}^{2}=0.32, \mathrm{~F}=38.13, \mathrm{df}=81, p<\right.$ 0.001) with the volumes increasing with time (Figure 1f-g).

\section{Discussion}

Our findings support the hypothesis that chronic patients have smaller brain volumes than first-episode patients and that there is an exponential relation between DoI and brain volume. The findings suggest that the volumetric differences between first-episode and chronic patients with schizophrenia is not uniform across different brain regions, but is selective to the frontal lobe, the parieto-occipital cortex and putamen. More interestingly, there is an increase in the premotor cortical volume with duration of illness, which suggests that different processes influencing both volumetric increase and decrease may be occurring concomitantly.

Our findings on PFC grey matter are consistent with that of Molina et al (2004) in three respects: (1) PFC grey matter was smaller in chronic patients compared with first-episode patients, (2) PFC grey matter was not smaller in FE patients compared with healthy controls, and (3) the association between DoI and brain volume across the patient groups was exponential. Our findings on PFC also support an earlier report of a greater rate of frontal lobe reduction over a 2.5 year duration in first-episode patients compared with previously-treated patients (Gur et al, 1998). 
A second finding was that the premotor cortex was larger in the chronic patients compared to the first-episode patients and that there was an exponential increase in volume with longer duration of illness. It may be suggest that the premotor cortex was enlarged secondary to neuroleptic medication. However, brain enlargement in schizophrenia has been typically reported in the basal ganglia as a result of neuroleptic medication (Bridle et al, 2002; Chakos et al, 1994; 1998; Corson et al, 1999; Gur et al, 1998). But, the supplementary motor area (SMA) sends projections to the basal ganglia and also receives projections from the basal ganglia via the thalamus (Frackowiak et al, 1997). It is therefore possible that the premotor cortex enlargement was secondary to that of putamen. Alternately, the volumetric increase in the premotor cortex may reflect an attempt to cope with a volumetric reduction of the PFC as the disease develops in an effort to take over some of the functions of the PFC.

The larger lateral ventricles in the chronic patients compared to first-episode patients, but the absence of a difference between their respective healthy control groups, complements the brain volume differences observed in the PFC and parieto-occipital grey matter. This finding is consistent with evidence for an increase in ventricular volume over time (DeLisi et al, 1998; 2004; 1997; 1995; Leiberman et al, 2001; Mathalon et al, 2001; Nair et al, 1997; Saijo et al, 2001).

One of the pathogenetic models of schizophrenia to explain MRI findings of progressive abnormality but the apparent absence of neurodegenerative changes from pathological studies is that of excessive neuronal apoptosis rather than necrosis. Pathophysiological studies of schizophrenia have reported the absence of gliosis (Pakkenberg, 1993; Selemon et al, 1998), 
whereas apoptosis, which is not associated with gliosis, can occur as a result of exogenous insult to the brain (Akbarian et al, 1996; Woods, 1998). A loss of neuropil due to excessive synaptic pruning might also cause decreased brain volume (Woods, 1998). Decreased neuropil and neuronal size, rather than neuronal loss have been observed in the hippocampus (Benes et al, 1991). Altered functioning of neurotransmitters might be taking place (Deakin \& Simpson, 1997b; Gluck et al, 2002). As reviewed by Deakin and Simpson (1997a), there is an asymmetric loss of glutamate terminals in the temporal lobe and reduced GABA function secondary to a loss of glutamatergic input. They suggest that the terminals that degenerate originate in the orbital frontal cortex that in turn sends afferents to the polar temporal cortex. These afferents are prone to degeneration because they may arise from cells that have not undergone normal postdevelopmental remodelling (Deakin \& Simpson, 1997b).

In conclusion, the present study provides evidence for a difference in brain volume between patients at two different stages of schizophrenia over and above that in healthy controls. It shows that this difference is not uniform across the brain, but that it is pronounced in the frontal and parieto-occipital and basal ganglial regions and directly related to the DoI. An interesting finding of a larger premotor cortical volume at a later stage of illness draws our attention to the potentiating effect of neuroleptic drugs.

\section{Acknowledgement}

Support for this research was provided by Grosvenor House Group and Psychmed Ltd. 


\section{References}

Akbarian, S., Kim, J. J., Potkin, S. G., Herrick, W. P., Bunney, W. E., Jr., \& Jones, E. G. (1996). Maldistribution of interstitial neurons in prefrontal white matter of the brains of schizophrenic patients. Archives of General Psychiatry 53, 425-436.

Bachmann, S., Bottmer, C., Pantel, J., Schroder, J., Amann, M., Essig, M., \& Schad, L. R. (2004). MRI-morphometric changes in first-episode schizophrenic patients at 14 months follow-up. Schizophrenia Research 67, 301-3.

Barta, P. E., Dhingra, L., Royall, R., \& Schwartz, E. (1997). Improving stereological estimates for the volume of structures identified in three-dimensional arrays of spatial data. Journal of Neuroscience Methods 75, 111-118.

Benes, F. M., Sorenson, I., \& Bird, E. D. (1991). Reduced neuronal size in posterior hippocampus of schizophrenic patietns. Schizophrenia Bulletin 17, 597-608.

Bilder, R. M., Wu, H., Bogerts, B., Degreef, G., Ashtari, M., Alvir, J. M., Snyder, P. J., \& Lieberman, J. A. (1994). Absence of regional hemispheric volume asymmetries in firstepisode schizophrenia. American Journal of Psychiatry. 151, 1437-1447.

Bridle, N., Pantelis, C., Wood, S. J., Coppola, R., Velakoulis, D., McStephen, M., Tierney, P., Le, T. L., Fuller, T. E., \& Weinberger, D. R. (2002). Thalamic and caudate volumes in monozygotic twins discordant for schizophrenia. Australian and New Zealand Journal of Psychiatry 36, 347-354.

Chakos, M. H., Lieberman, J. A., Bilder, R. M., Borenstein, M., Lerner, G., Bogerts, B., Wu, H., Kinon, B., \& Ashtari, M. (1994). Increase in caudate nuclei volumes of first-episode schizophrenic patients taking antipsychotic drugs. American Journal of Psychiatry 151, 1430-1436.

Chakos, M. H., Shirakawa, O., Lieberman, J. A., Lee, H., Bilder, R. M., \& Tamminga, C. A. (1998). Striatal enlargement in rats chronically treated with neuroleptic. Biological Psychiatry 44, 675-684.

Copolov, D., Velakoulis, D., McGorry, P., Mallard, C., Yung, A., Rees, S., Jackson, G., Rehn, A., Brewer, W., \& Pantelis, C. (2000). Neurobioloigcal findings in prodromal and early phase schizophrenia. Brain Research Reviews 31, 157-165.

Corson, P. W., Nopoulos, P., Miller, D. D., Arndt, S., \& Andreasen, N. C. (1999). Change in basal ganglia volume over 2 years in patients with schizophrenia: typical versus atypical neuroleptics. American Journal of Psychiatry 156, 1200-1204.

Deakin, J. F., \& Simpson, M. D. (1997a). A two-process theory of schizophrenia: evidence from studies in post-mortem brain. Journal of Psychiatric Research 31, 277-295.

Deakin, J. F., \& Simpson, M. D. (1997b). A two-process theory of schizophrenia: evidence from studies in post-mortem brain. Journal of Psychiatric Research. 31, 277-95. 
DeLisi, L. E., \& Hoff, A. L. (2005). Failure to find progressive temporal lobe volume decreases 10 years subsequent to a first episode of schizophrenia. Psychiatry Research 138, 265-8.

DeLisi, L. E., Sakuma, M., Ge, S., \& Kushner, M. (1998). Association of brain structural change with the heterogeneous course of schizophrenia from early childhood through five years subsequent to a first hospitalization. Psychiatry Research 84, 75-88.

DeLisi, L. E., Sakuma, M., Maurizio, A. M., Relja, M., \& Hoff, A. L. (2004). Cerebral ventricular change over the first 10 years after the onset of schizophrenia. Psychiatry Research 130, 57-70.

DeLisi, L. E., Sakuma, M., Tew, W., Kushner, M., Hoff, A. L., \& Grimson, R. (1997). Schizophrenia as a chronic active brain process: A study of progressive brain structural change subsequent to the onset of schizophrenia. Psychiatry Research: Neuroimaging 74, 129-140.

DeLisi, L. E., Tew, W., Xie, S., Hoff, A. L., Sakuma, M., Kushner, M., Lee, G., Shedlack, K., Smith, A. M., \& Grimson, R. (1995). A prospective follow-up study of brain morphology and cognition in first-episode schizophrenic patients: preliminary findings. Biological Psychiatry. 38, 349-60.

Dickey, C. C., Salisbury, D. F., Nagy, A. I., Hirayasu, Y., Lee, C. U., McCarley, R. W., \& Shenton, M. E. (2004). Follow-up MRI study of prefrontal volumes in first-episode psychotic patients. Schizophrenia Research 71, 349-51.

Ettinger, U., Chitnis, X. A., Kumari, V., Fannon, D. G., Sumich, A. L., O'Ceallaigh, S., Doku, V. C., \& Sharma, T. (2002). Magnetic resonance imaging of the thalamus in first-episode psychosis. American Journal of Psychiatry 158, 116 - 118.

Fannon, D., Chitnis, X., V., D., Tennakoon, L., O'Ceallaigh, S., Soni, W., Sumich, A., Lowe, J., Santamaria, M., \& Sharma, T. (2000). Features of structural brain abnormality detected in first-episode psychosis. American Journal of Psychiatry 157, 1829-1834.

First, M. B., Spitzer, R. L., Gibbon, M., \& Williams, J. B. (1996b). Structured Clinical Interview for DSM-IV Axis 1 Disorders - Non-Patient Edition (SCID-I/NP), version 2.0. New York, New York State Psychiatric Institute, Biometrics Research.

Frackowiak, R. S., Friston, K. J., Frith, C. D., \& Dolan, R. J. (1997). Human Brain Function. San Diego: Academic Press.

Gluck, M. R., Thomas, R. G., Davis, K. L., \& Haroutunian, V. (2002). Implications for altered glutamate and GABA metabolism in the dorsolateral prefrontal cortex of aged schizophrenic patients. American Journal of Psychiatry 159, 1165-1173.

Gur, R. E., Cowell, P., Turetsky, B. I., Gallacher, F., Cannon, T., Bilker, W., \& Gur, R. C. (1998). A follow-up magnetic resonance imaging study of schizophrenia. Relationship of neuroanatomical changes to clinical and neurobehavioral measures. Archives of General Psychiatry 55, 145-152. 
Harrison, P. J. (1999). The neuropathology of schizophrenia. A critical review of the data and their interpretation. Brain. 122, 593-624.

James, A. C. D., Javaloyes, A., James, S., \& Smith, D. M. (2002). Evidence for non-progressive changes in adolescent-onset schizophrenia: Follow-up magnetic resonance imaging study. British Journal of Psychiatry 180, 339-344.

Jaskiw, G. E., Juliano, D. M., Goldberg, T. E., Hertzman, M., Urow-Hamell, E., \& Weinberger, D. R. (1994). Cerebral ventricular enlargement in schizophreniform disorder does not progress. A seven year follow-up study. Schizophrenia Research 14, 23-28.

Kraepelin, E. (1899/1989). Psychiatry: a textbook for students and physicians. New Delhi: Amerind Publishing Co.

Kraepelin, E. (1919/1971). Dementia Praecox. New York: Churchill Livingstone Inc.

Leiberman, J., Chakos, M., Wu, H., Alvir, J., Hoffman, E., Robinson, D., \& Bilder, R. (2001). Longitudinal study of brain morphology in first episode schizophrenia. Biological Psychiatry 49, 487-499.

Mathalon, D. H., Sullivan, E. V., Lim, K. O., \& Pfefferbaum, A. (2001). Progressive brain volume changes and the clinical course of schizophrenia in men: a longitudinal magnetic resonance imaging study. Archives of General Psychiatry 58, 148-157.

Molina, V., Sanz, J., Sarramea, F., Benito, C., \& Palamo, T. (2004). Lower prefrontal gray matter volume in schizophrenia in chronic but not in first episode schizophrenia patients. Psychiatry Research: Neuroimaging 131, 45-56.

Nair, T. R., Christensen, J. D., Kingsbury, S. J., Kumar, N. G., Terry, W. M., \& Garver, D. L. (1997). Progression of cerebroventricular enlargement and the subtyping of schizophrenia. Psychiatry Research 74, 141-150.

Pakkenberg, B. (1993). Total nerve cell number in neocrotex in chronic schizophrenics and controls estimated using optical dissectors. Biological Psychiatry 34, 768-772.

Portas, C. M., Goldstein, J. M., Shenton, M. E., Hokama, H. H., Wible, C. G., Fischer, I., Kikinis, R., Donnino, R., Jolesz, F. A., \& McCarley, R. W. (1998). Volumetric evaluation of the thalamus in schizophrenic male patients using magnetic resonance imaging. Biological Psychiatry 43, 649-659.

Puri, B. K., Hutton, S. B., Saeed, N., Oatridge, A., V., H. J., Duncan, L., Chapman, M. J., Barnes, T. R., Bydder, G. M., \& Joyce, E. M. (2001). A serial longitudinal quantitative MRI study of cerebral changes in first-episode schizophrenia using image segmentation and subvoxel registration. Psychiatry Research 106, 141-150.

Saijo, T., Abe, T., Someya, Y., Sassa, T., Sudo, Y., Suhara, T., Shuno, T., Asai, K., \& Okubo, Y. (2001). Ten year progressive ventricular enlargement in schizophrenia: an MRI morphometrical study. Psychiatry \& Clinical Neurosciences. 55, 41-47. 
Selemon, L. D., Rajkowska, G., \& Goldman-Rakic, P. S. (1998). Elevated neuronal density in prefrontal area 46 in brains from schizophrenic patients: application of a threedimensional, sterological counting method. Journal of Comprehensive Neurology 392, 402-412.

Spitzer, R. L., Williams, J. B. W., \& Gibbon, M. (1995). Structured Clinical Interview for DSMIV (SCID). New York: New York State Psychiatric Institute, Biometrics Research.

Stephanis, N., Frangou, S., Yekeley, J., Sharma, T., O'Connell, P., Morgan, K., Sigmudsson, T., Taylor, M., \& Murray, R. M. (1999). Hippocampal volume reduction in schizophrenia: effects of genetic risk and pregnancy and birth complications. Biological Psychiatry 46, 697-702.

Stevens, J. R. (1991). Schizophrenia: Static or progressive pathophysiology? Schizophrenia Research 5, 184-186.

Sumich, A., Chitnis, X. A., Fannon, D. G., O'Ceallaigh, S., Doku, V. C., Falrowicz, A., Marshall, N., Matthew, V. M., Potter, M., \& Sharma, T. (2002). Temporal lobe abnormalities in first-episode psychosis. American Journal of Psychiatry 159, 1232-1234.

Weinberger, D. R., \& McClure, R. K. (2002). Neurotoxicity, neuroplasticity, and magnetic resonance imaging morphometry: What is happening in the schizophrenic brain? Archives of General Psychiatry 59, 553-558.

Woods, B. T. (1998). Is schizophrenia a progressive neurodevelopmental disorder? American Journal of Psychiatry 155, 1661-1670.

Woods, B. T., Yurgelun-Todd, D., Goldstein, J. M., Seidman, L. J., \& Tsuang, M. T. (1996). MRI brain abnormalities in chronic schizophrenia: one process or more? Biological Psychiatry 40, 585-596. 
Table 1. Demographic characteristics of participants.

\begin{tabular}{|c|c|c|c|c|c|c|}
\hline & $\begin{array}{l}\text { FE patients } \\
\qquad(\mathrm{n}=34)\end{array}$ & $\begin{array}{l}\text { FE-matched } \\
\text { controls } \\
(\mathrm{n}=18)\end{array}$ & $\begin{array}{c}\text { CH patients } \\
(\mathrm{n}=49)\end{array}$ & $\begin{array}{c}\text { CH-matched } \\
\text { controls } \\
(\mathrm{n}=21)\end{array}$ & $\chi^{2}$ & df \\
\hline$\ddagger$ Age, yrs - mean (SD) & $23.71(4.96)$ & $25.56(5.44)$ & $40.12(10.24)$ & $36.19(11.27)$ & $56.07 *$ & 3 \\
\hline †Education, yrs - mean (SD) & $12.06(1.79)$ & $16.06(1.66)$ & $12.88(2.68)$ & $14.86(3.10)$ & $35.08^{*}$ & 3 \\
\hline$†$ Gender ( $\%$ male $)$ & 70.60 & 66.70 & 65.30 & 71.40 & 0.40 & 3 \\
\hline$\dagger$ Handedness (\% right-handed) & 91.20 & 88.88 & 93.90 & 100 & 2.38 & 3 \\
\hline$\S$ DoI (months) & $7.79(7.65)$ & - & $169.90(112.03)$ & - & $-10.10^{*}$ & 48.64 \\
\hline
\end{tabular}

$\ddagger$ Kruskal-Wallis test performed; $\dagger \chi^{2}$ test performed; ${ }^{\S}$ t-test assuming unequal variance; FE first-episode, $\mathrm{CH}$ chronic

$* \mathrm{p}<0.05$ 
Table 2. Means (S.D.) for region of interest volumes (cubic $\mathrm{cm}$ ) in first-episode patients, chronic patients and matched healthy controls

\begin{tabular}{lccccc}
\hline & FE Patient & FE Control & CH patient & CH control & \\
Brain region & $(\mathrm{n}=34)$ & $(\mathrm{n}=18)$ & $(\mathrm{n}=49)$ & $(\mathrm{n}=21)$ & Univariate F \\
\hline Whole brain & $836.62(72.18)$ & $903.86(92.76)$ & $820.36(125.87)$ & $855.76(115.95)$ & 0.003 \\
Cortical grey matter & $463.64(44.33)$ & $502.46(57.24)$ & $420.06(72.21)$ & $460.96(59.42)$ & 2.85 \\
& & & & & \\
Total lateral ventricle & $9.83(5.28)$ & $8.38(3.28)$ & $13.46(5.51)$ & $9.43(4.61)$ & $6.35^{*}$ \\
Total PFC & $110.93(15.36)$ & $118.05(18.74)$ & $97.51(19.63)$ & $111.94(21.94)$ & $5.25^{*}$ \\
Grey matter of PFC & $70.95(10.42)$ & $74.66(13.89)$ & $57.19(11.45)$ & $67.76(10.36)$ & $11.49^{*}$
\end{tabular}




\begin{tabular}{|c|c|c|c|c|c|}
\hline Total SMC & $221.37(30.31)$ & $225.54(39.87)$ & $216.98(37.38)$ & $223.21(30.12)$ & 0.20 \\
\hline Grey matter of SMC & $104.75(23.44)$ & $100.51(16.08)$ & $95.07(20.90)$ & $93.31(20.81)$ & 0.94 \\
\hline Total POC & $231.65(28.64)$ & 269.79 (33.67) & $223.83(36.63)$ & $248.28(44.24)$ & 0.07 \\
\hline Grey matter of POC & $147.12(20.65)$ & $165.14(19.66)$ & $128.90(25.27)$ & $152.61(25.27)$ & $4.92 *$ \\
\hline Total temporal lobe & $101.49(16.03)$ & $112.68(17.65)$ & $100.57(21.52)$ & $99.23(19.34)$ & 0.82 \\
\hline Grey matter of T.lobe & $72.16(10.67)$ & $82.06(12.18)$ & $66.62(12.94)$ & $66.09(13.97)$ & 0.01 \\
\hline Cerebellum & $101.26(10.04)$ & $107.68(13.77)$ & $103.14(16.55)$ & $100.20(11.56)$ & 1.93 \\
\hline Thalamus & $11.91(1.35)$ & $12.57(1.25)$ & $10.90(1.52)$ & $11.07 \quad(1.22)$ & 0.74 \\
\hline Hippocampus $^{\mathrm{a}}$ & $4.39(0.50)$ & $5.04(0.45)$ & $3.97(0.92)$ & $4.63(0.57)$ & -1.29 \\
\hline Putamen $^{\mathrm{a}}$ & $5.13(0.90)$ & $4.44(0.68)$ & $7.41(1.86)$ & $5.92(1.16)$ & $-5.80^{*}$ \\
\hline
\end{tabular}


${ }^{a}$ Wilcoxon Signed Ranks test performed due to heterogeneity of variance; PFC: Prefrontal cortex; PMC: Premotor cortex; POC:

Parieto-occipital cortex; SMC: Supplementary-motor cortex; T.lobe: Temporal lobe

$* \mathrm{p}<0.05$ 
Figure 1. Plot of duration of illness (in months) and volume of brain regions of interest (in cubic cm) with logarithmic fit line

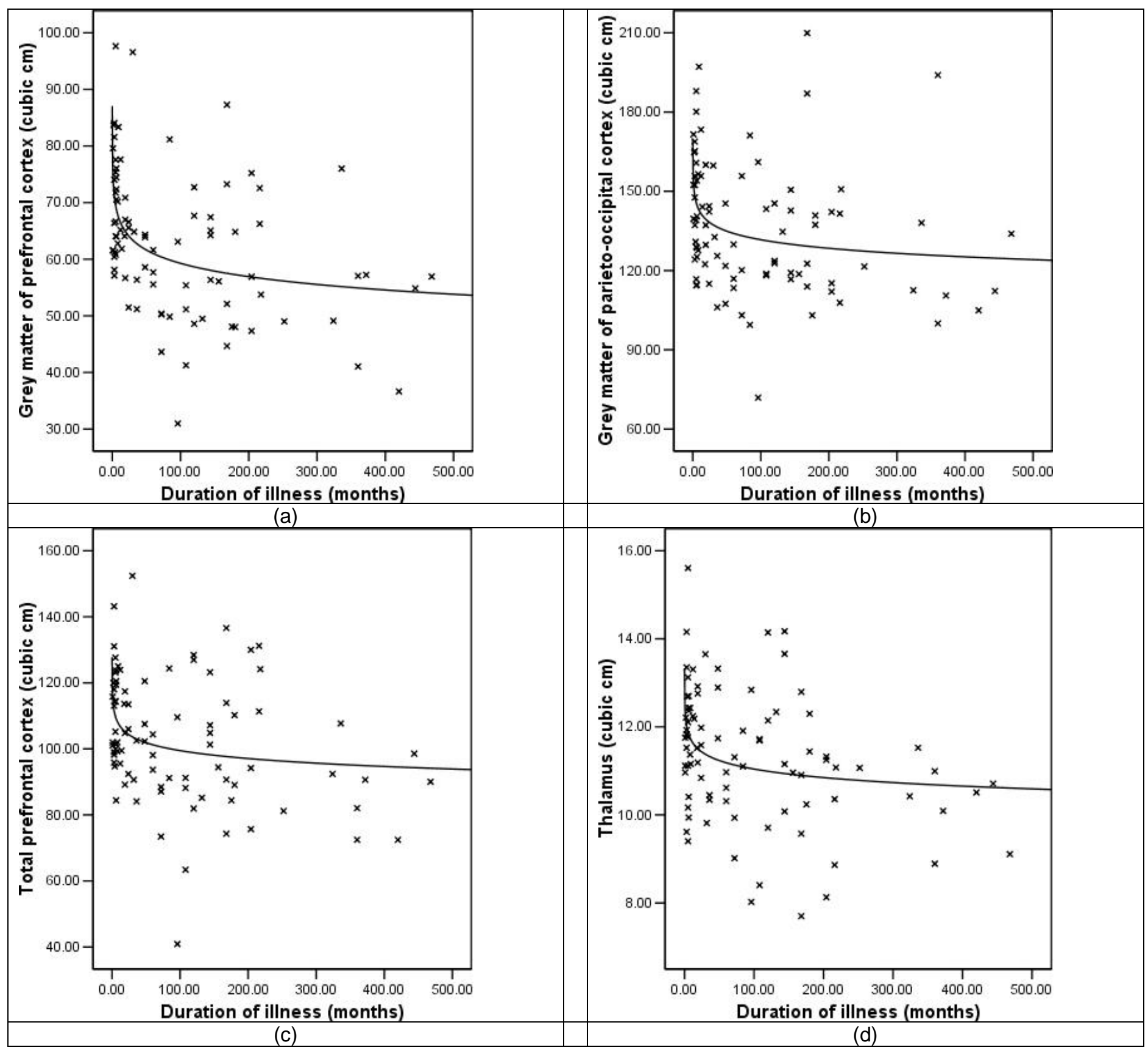




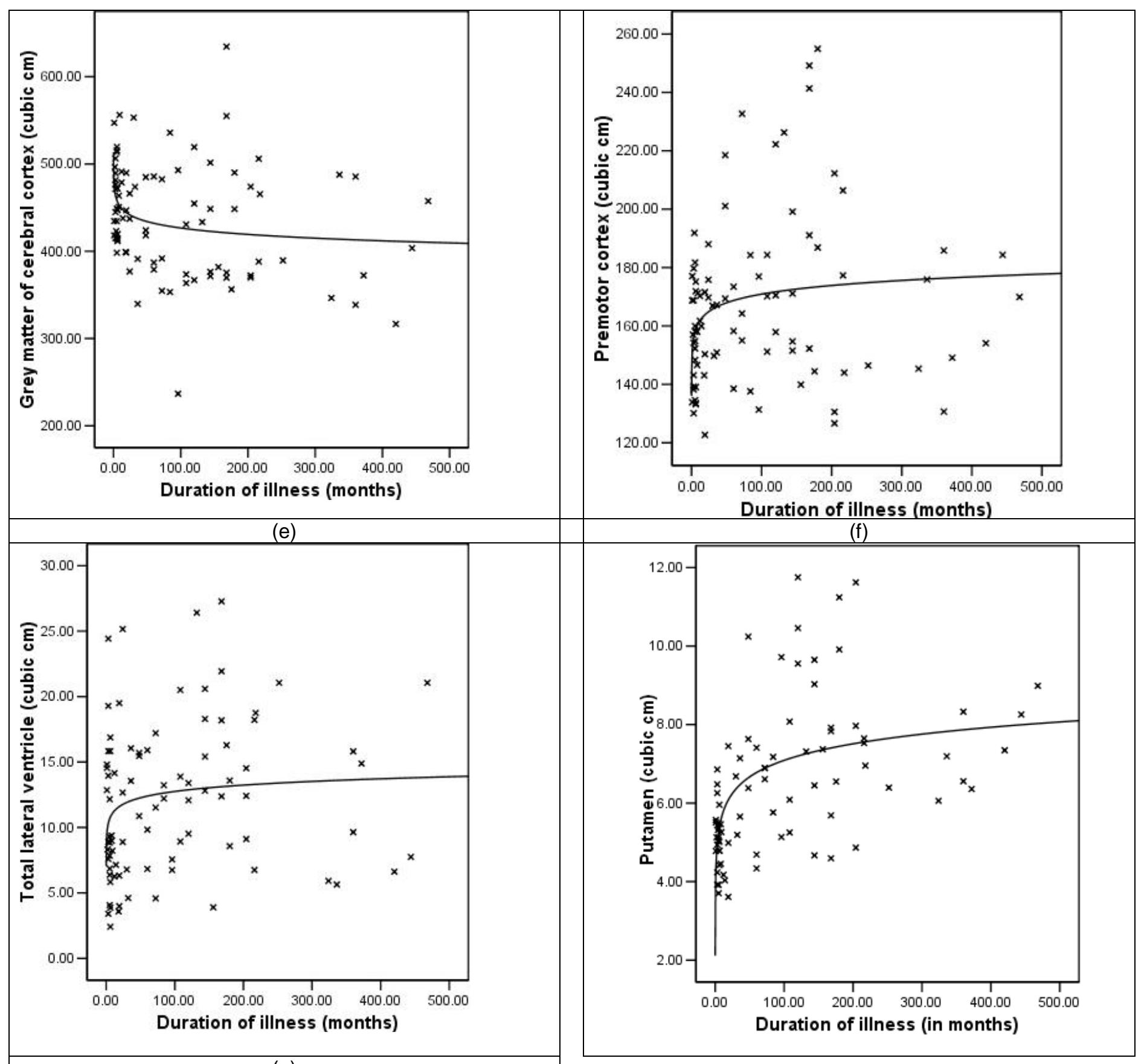

(g) 